\title{
Crossing mitochondrial barriers
}

A large number of diseases (as well as ageing) are associated with mitochondrial dysfunction, and over 200 pathogenic mitochondrial DNA mutations have so far been identified. There is no treatment for these diseases, and many of them are fatal. Gene therapy could be an attractive option; however, delivery of genes across the double membrane of mitochondria is particularly challenging. Reporting in PNAS, John Guy and colleagues now describe the development of a mitochondriatargeted adeno-associated virus (AAV)-based gene vector and its use in experimental models of Leber's hereditary optic neuropathy (LHON) - a form of blindness that is caused by point mutations in mitochondrial genes.

Most cases of LHON are associated with mutations in one of three genes that encode subunits of complex I, which forms part of the mitochondrial respiratory chain, resulting in reduced ATP synthesis. Half of all patients with LHON carry a particular point mutation in $\mathrm{NADH}$ dehydrogenase subunit 4 (ND4).

To design a vector capable of delivering a wild-type human ND4 gene into mitochondria, the gene which can only be translated in mitochondria - was linked to a mitochondrial promoter and cloned into the AAV backbone. This plasmid was packaged into an AAV capsid containing the AAV protein VP2 fused to a mitochondrial targeting sequence (MTS). In vitro experiments showed that the resulting vector was capable of delivering ND4 to mitochondria and restoring ATP synthesis.

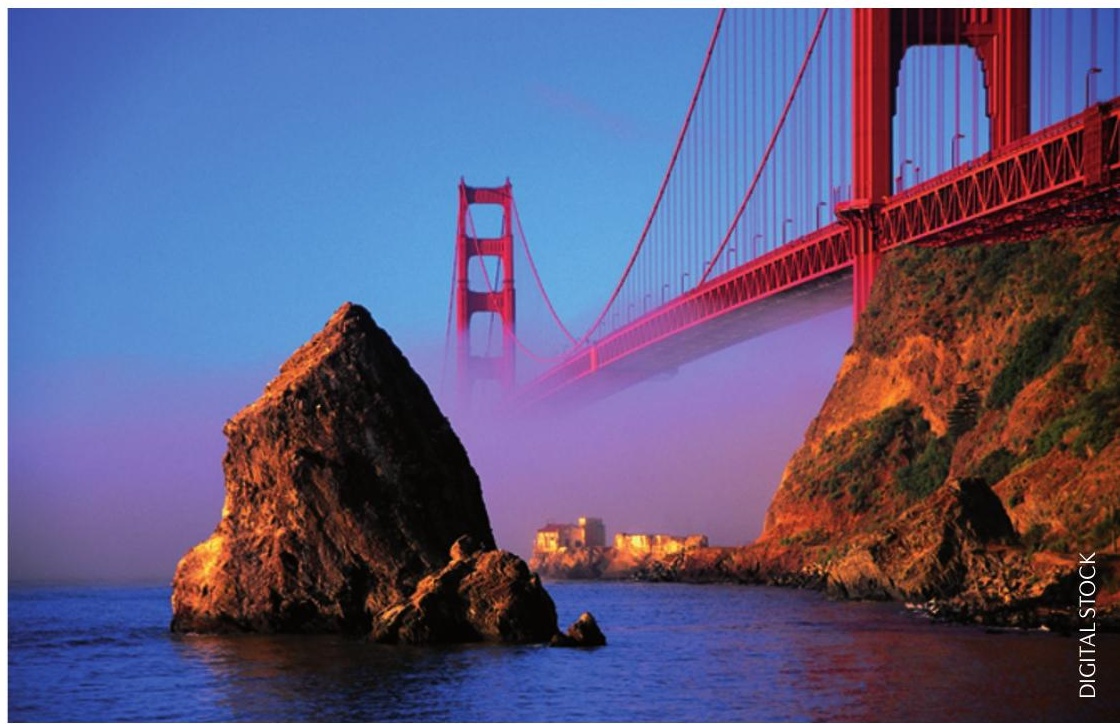

To test the potential for in vivo delivery of genes to mitochondria, the authors injected the construct into the vitreous body of mouse eyes, using vectors that lacked either the MTS or ND4 as controls. It was shown that the ND4 protein is still expressed 6 months after injection, and studies suggested that the human ND4 gene is integrated into the mitochondrial genome and replicated along with mitochondrial DNA.

Next, the construct was tested in a model of LHON. As there is no transgenic animal model with mutated ND4, the authors used the same vector described above, but with mutated rather than wild-type ND4, to induce disease. A pattern electroretinogram, reflecting visual capability, showed that eyes injected with wild-type ND4, followed 2 days later by mutant ND4, had normal visual capability, whereas injection with the control vector and then with mutated ND4 impaired vision. Furthermore, treatment with wild-type ND4 also prevented optic atrophy, demonstrating that mitochondria-targeted delivery of ND4 ameliorates the functional consequences of the mutated ND4 homolog.

These experiments show that mitochondrial targeting for transgene delivery is possible. As the AAV vector can accommodate genes of up to $5 \mathrm{~kb}$ in length, the authors suggest that it might provide a platform for the introduction of almost any mitochondrial gene, possibly even allowing the insertion of large-kilobase deletions that are associated with ageing, back into the adult organelle.

\section{Alexandra Flemming}

ORIGINAL RESEARCH PAPER Yu, H. et al. Gene delivery to mitochondria by targeting modified adenoassociated virus suppresses Leber's hereditary optic neuropathy in a mouse model. Proc. Natl Acad. Sci. USA 20 Apr 2012 (doi:10.1073/pnas.1119577109) 